$04 ; 13$

\title{
Распыление вольфрама ионами бериллия и неона
}

\author{
(ㄱ Д.С. Мелузова, П.Ю. Бабенко, А.Н. Зиновьев, А.П. Шергин \\ Физико-технический институт им. А.Ф. Иоффе РАН, Санкт-Петербург, Россия \\ E-mail: dmeluzova@gmail.com
}

Поступило в Редакцию 29 июля 2020 г.

В окончательной редакции 11 сентября 2020 г.

Принято к публикации 12 сентября 2020 г.

Методом молекулярной динамики рассчитаны коэффициенты распыления и их угловые зависимости при бомбардировке вольфрама ионами Ве и $\mathrm{Ne}$. В случае $\mathrm{Ne}$ имеется хорошее согласие с экспериментом. Полученные данные для случая $\mathrm{Be}-\mathrm{W}$ нужны для расчетов поступления примесей при бомбардировке ионами Ве материала дивертора — вольфрама в плазме токамака-реактора ИТЭР. Предложена модель, объясняющая универсальность поведения коэффициентов распыления в припороговой области при бомбардировке вольфрама легкими ионами.

Ключевые слова: распыление, бериллий, вольфрам, ИТЭР.

DOI: 10.21883/PJTF.2020.24.50422.18487

В качестве материала дивертора в токамаке ИТЭР планируется использовать вольфрам, а в качестве материала первой стенки - бериллий. Как показано в работах $[1,2]$, атомы изотопов водорода, покидающие плазму, бомбардируют стенку, что приводит к заметному поступлению Ве в плазму. Концентрация Ве может достигать $2-4 \%$ от плотности плазмы. Двигаясь по сепаратрисе, атомы Ве ионизуются до ядер и, ускоряясь потенциалом плазма-стенка до энергий 300-800 eV [1], без заметного поглощения проходят слой диверторной плазмы и вызывают распыление дивертора. Поступление вольфрама в плазму заметно меняет характеристики разряда [3-6]. Если концентрация вольфрама достигнет летального значения $0.1 \%$, всю энергию плазмы будет уносить излучение ионов примеси, и нужная температура не будет достигнута. В настоящее время отсутствуют экспериментальные данные о распылении вольфрама ионами бериллия. В ряде работ рассчитывается распыление вольфрама и различных химических соединений с вольфрамом [7-9] и моделируются потоки бериллия в плазме $[2,10]$. Получение данных о коэффициентах распыления вольфрама и их угловых зависимостях также является предметом интенсивных теоретических и экспериментальных исследований [6,11-13]. В задачу настоящей работы входило провести расчеты распыления вольфрама ионами Ве и $\mathrm{Ne}$. В случае $\mathrm{Ne}$ имеются надежные экспериментальные данные, что позволяет проверить правильность расчета.

Был разработан код для расчета распыления мишени из аморфного вольфрама. Расчеты коэффициента проводятся методом Монте-Карло с использованием принципов молекулярной динамики. Для описания взаимодействия между атомами мишени используется многочастичный потенциал, полученный в рамках модели погруженного атома в работе [14], с поправкой, учитывающей взаимодействие на близком расстоянии в соответствии c [15]. Для описания взаимодействия между налетающим атомом и атомами мишени использовался потенциал из работы [16], рассчитанный в рамках теории функционала плотности. Атомная структура мишени выстроена как идеальное аморфное тело с использованием кода [17]. Расстояние между атомами выбрано так, чтобы плотность моделируемой мишени соответствовала реальной плотности вольфрама. Мишень представляет собой полусферу, состоящую из $\sim 0.5 \cdot 10^{6}$ атомов. Бомбардировке подвергается ее плоская часть, а на оставшуюся поверхность наложены жесткие граничные условия. В изучаемом энергетическом диапазоне (до $1 \mathrm{keV}$ ) атомы мишени не приобретают достаточную энергию для образования каскадов движущихся атомов, поэтому распыление проходит преимущественно в режиме прямого выбивания, что позволяет учитывать подвижность только малой доли атомов мишени.

Как уже упоминалось, для случая бомбардировки вольфрама неоном есть экспериментальные данные [18] (приведены на рис. 1, $a$ ).

Треугольниками на рисунке показаны результаты нашего расчета методом молекулярной динамики (MD). Видно хорошее согласие с экспериментальными данными. Также приведен расчет с помощью предложенной нами модели обратного распыления (back scattering sputtering, BSS), речь о которой пойдет далее. Расчет по формуле Ямамуры $[19,20]$ дает заниженные значения коэффициента распыления и порога распыления. Программа ACAT [21] занижает порог распыления и завышает величину коэффициента распыления в области малых энергий. Программа SRIM [22] завышает величину порога распыления.

Точные данные о распылении вольфрама атомами бериллия представляют большой интерес для термоядерных исследований, но экспериментальные данные о коэффициентах распыления в литературе отсутствуют. Рассчитанные нами для комбинации $\mathrm{Be}-\mathrm{W}$ коэффициенты распыления в зависимости от энергии налетающих частиц представлены на рис. $1, b$. Имеется хорошее согласие наших данных с расчетами методом молеку- 

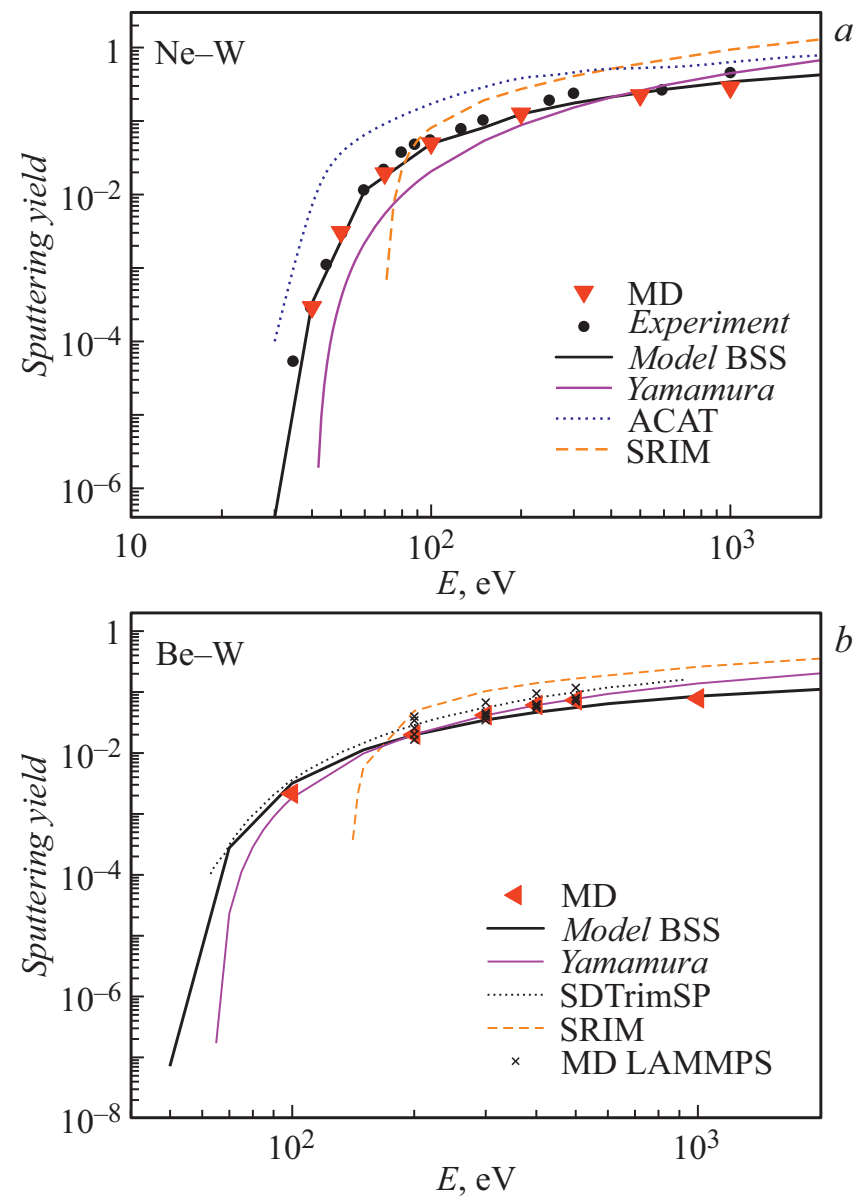

Рис. 1. Зависимости коэффициента распыления вольфрама от энергии бомбардирующих ионов неона $(a)$ и бериллия $(b)$.

лярной динамики программой LAMMPS из работы [23]. Также приведен расчет с использованием программы SDTrimSP из работы [6].

Для системы $\mathrm{Ne}-\mathrm{W}$ нами также была рассчитана зависимость коэффициента распыления от угла падения при бомбардировке ионами с начальной энергией $200 \mathrm{eV}$ и $1 \mathrm{keV}$. Результат представлен на рис. 2, $a$. Имеется хорошее согласие с расчетами Экштайна [24] с использованием программы TrimSP. Аналогичные зависимости получены также для бомбардировки вольфрама бериллием (рис. 2,b). В этом случае в литературе данные для сравнения отсутствуют. Зависимость для обеих исследованных систем имеет пик, который приходится на величину угла в интервале $60-80^{\circ}$ от нормали, что находится в согласии с теоретическими представлениями о характере данной зависимости $[19,20]$.

Для анализа данных о распылении вольфрама легкими атомами можно предложить модель выбивания поверхностных атомов потоком обратно рассеянных частиц (BSS). Рассмотрим рассеяние частицы массой $M_{1}$ на частице верхнего слоя твердого тела с массой $M_{2}$. Введем систему координат, направив ось $x$ по направлению проекции скорости налетающей частицы на поверхность твердого тела, а ось $z-$ перпендикулярно ему. Пучок частиц облучает площадку на поверхности с размерами $d \times d$, где $d-$ среднее расстояние между атомами поверхности, для случая $\mathrm{W} d=2.48 \AA$. Введем вектор $\mathbf{R}$, соединяющий точку вылета частицы из предпоследнего слоя (координаты $(0,0,0))$ и положение облучаемого атома вольфрама $\left(x_{1}, y_{1}, d\right)$. Координата $x_{1}$ меняется в пределах $(d \sin \theta-d / 2, d \sin \theta+d / 2)$, а координата $y_{1}$ в пределах $(-d / 2, d / 2)$.

Вектор скорости $\mathbf{v}$ имеет компоненты $(v \sin \theta, 0$, $v \cos \theta)$. Используя векторное произведение, получаем положение вектора $\mathbf{N}=[\mathbf{V} \times \mathbf{R}]$, перпендикулярного плоскости рассеяния. Величина вектора $N=v R \sin \alpha$, где $v$ и $R$ - модули соответствующих векторов, а $\alpha-$ угол между векторами. Значение прицельного параметра $\rho$ можно определить исходя из соотношения $\rho=R \sin \alpha=N / v$. С помощью известных формул классической механики можно рассчитать значение угла рассеяния $\chi$ в системе центра масс, соответствующее рассеянию при параметре удара $\rho$. Как известно, покоящаяся до столкновения частица с массой $M_{2}$ вылетает под углом $\theta_{2}=(\pi / 2-\chi / 2)$ в плоскости рассеяния. Введем нормализованные векторы $\mathbf{e}_{1}=(1 / v) V$,
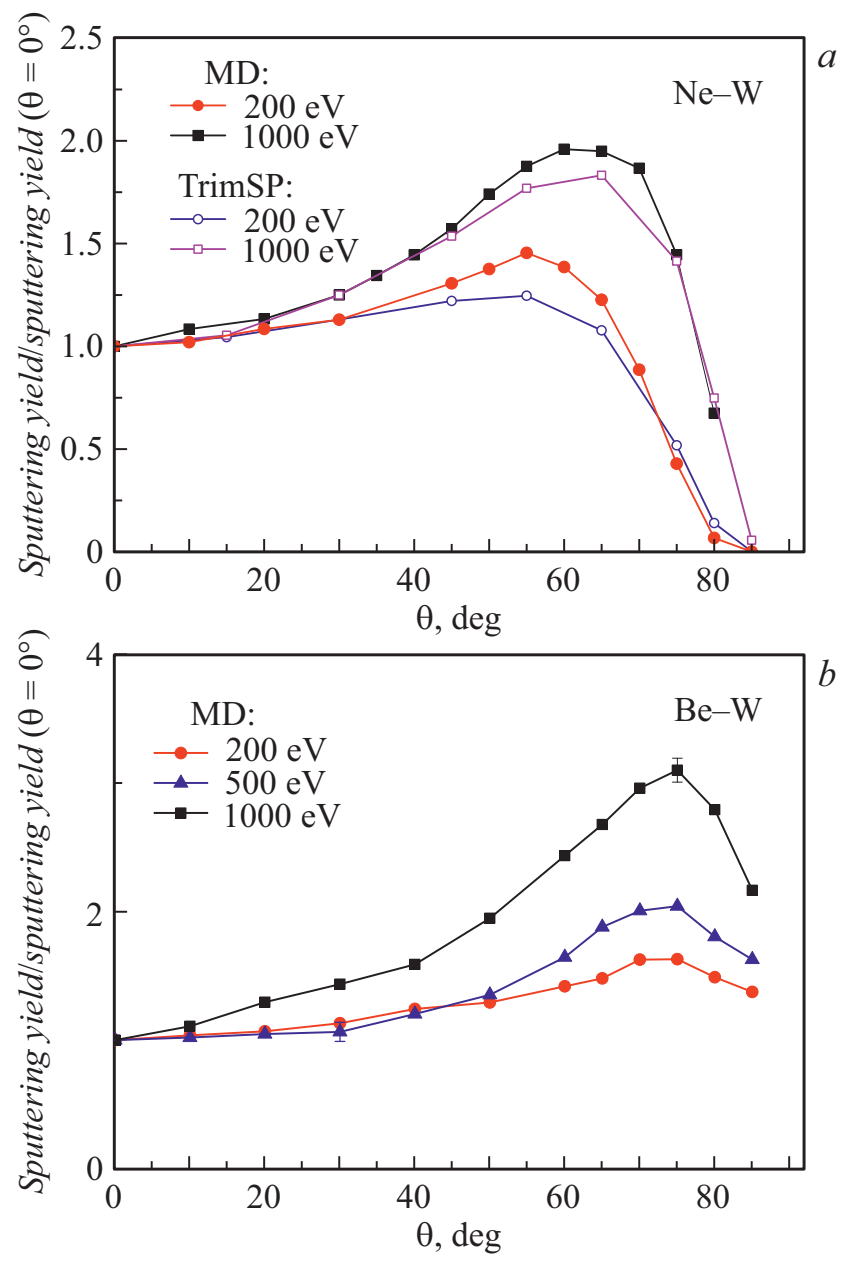

Рис. 2. Зависимости коэффициента распыления вольфрама ионами неона $(a)$ и бериллия $(b)$ от угла падения пучка. Угол отсчитывается от нормали к поверхности. 
$\mathbf{e}_{2}=1 /(\rho v) \mathbf{N}$ и вектор $\mathbf{e}_{3}=\left[\mathbf{e}_{1} \times \mathbf{e}_{2}\right]$. Частица отдачи после рассеяния имеет компоненту скорости $v_{2} \cos \theta_{2}$ вдоль вектора е 1 и компоненту $v_{2} \sin \theta_{2}$ вдоль вектоpa $\mathbf{e}_{3}$. Таким образом, компонента скорости частицы отдачи вдоль оси $z$ может быть вычислена по формуле $v_{2 z}=v_{2} \cos \theta_{2} e_{1 z}+v_{2} \sin \theta_{2} e_{3 z}$.

Используя соотношения $\theta_{2}=(\pi / 2-\chi / 2), e_{1 z}=\cos \theta$, получаем

$$
v_{2 z}=v_{2} \sin (\chi / 2) \cos \theta+\cos (\chi / 2) \sin \theta e_{2 y} .
$$

Величина $v_{2}$ связана со скоростью соударения $v$ соотношением $v_{2}=2 M_{1} v /\left(M_{1}+M_{2}\right) \sin (\chi / 2)$. При лобовом ударе частица массой $M_{1}$ передает частице массой $M_{2}$ энергию, равную

$$
E_{t h}=\frac{4 M_{1} M_{2}}{\left(M_{1}+M_{2}\right)^{2}} \sin ^{2}\left(\frac{\chi}{2}\right)>E_{s},
$$

где $\chi-$ угол рассеяния в системе центра масс, $E_{s}-$ энергия сублимации. При $\chi=\chi_{t h}$ имеем $E_{t h}=E_{s}$. Для распыления материала компонента энергии, переданная атому вольфрама вдоль оси $z$, должна превышать энергию сублимации $E_{s}$, равную $8.45 \mathrm{eV}$ для вольфрама. Как видно из этой формулы, при лобовом соударении легкая частица передает атому вольфрама малую часть своей энергии, и пороговая энергия составляет для случая $\mathrm{Ne}-\mathrm{W} E_{t h}=24 \mathrm{eV}$, для случая $\mathrm{Be}-\mathrm{W} E_{t h}=48 \mathrm{eV}$. Таким образом, рассчитав энергетическое и угловое распределение обратно рассеявшихся первичных частиц и сканируя пучком по площадке, упомянутой ранее, можно отобрать число случаев, когда соударение передает атому материала вдоль оси $z$ энергию, превышающую энергию сублимации. Нормируя число распыленных атомов на число первоначально падающих частиц, получаем коэффициент распыления.

Частицы с энергией меньше порога не вносят вклад в распыление. Средняя энергия обратно рассеянных частиц, вносящих вклад в распыление, составляет примерно $50-60 \%$ от начальной энергии бомбардирующих частиц, что приводит к соответствующему сдвигу порога распыления.

Поскольку в распыление может вносить вклад несколько поверхностных слоев вольфрама, следует учесть фактор $R_{p} / d_{l}$, где $R_{p}-$ проективный пробег, a $d_{l}-$ среднее расстояние между слоями вольфрама. На рис. 1 приведено сопоставление значения коэффициента распыления, рассчитанного по указанной модели, с экспериментом и расчетом методом МД. Имеется неплохое согласие. Преимущество данной модели возможность оценки коэффициента распыления без трудоемких расчетов. Модель неприменима при распылении материала ионами с массой, сравнимой с массой атомов мишени, когда существенна роль каскадных процессов, и распылении под малыми углами, когда нужно учитывать взаимодействие налетающей частицы с совокупностью атомов мишени.

На рис. 3 представлены зависимости коэффициентов распыления вольфрама ионами $\mathrm{D}, \mathrm{He}, \mathrm{Be}, \mathrm{Ne}$. Для $\mathrm{Be}$

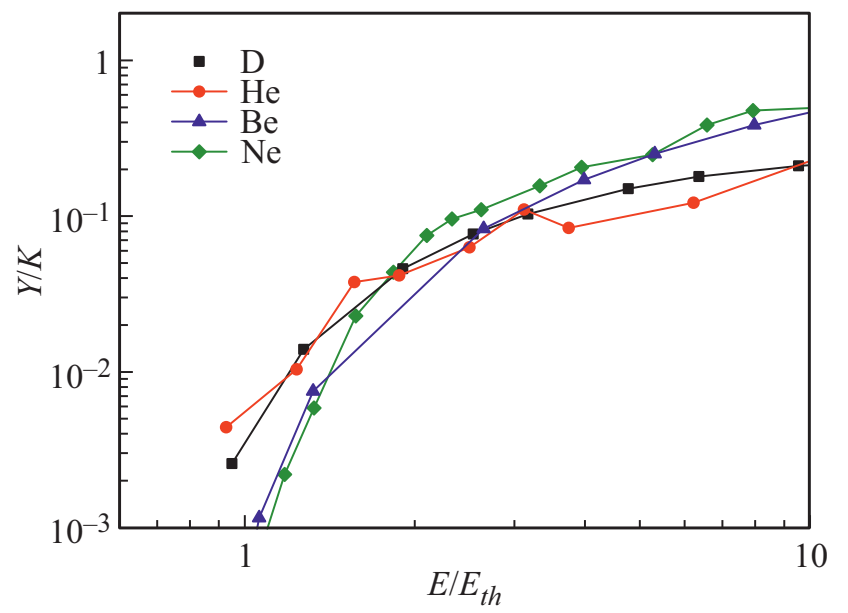

Рис. 3. Зависимость коэффициента распыления вольфрама различными ионами в приведенных координатах.

использованы расчетные данные (MD), в остальных случаях - экспериментальные. Шкала энергий нормирована на величины пороговой энергии. Для нормирования по абсолютной шкале использован коэффициент $K=\sigma\left(\chi_{t h}\right) / d_{l}^{2}$ при $E / E_{t h}=4$. Здесь $\sigma\left(\chi_{t h}\right)-$ сечение рассеяния на угол, больший $\chi_{t h}$. Коэффициент $K$ можно трактовать как вероятность выбивания атома вольфрама потоком обратно рассеянных частиц.

Как видно из рис. 3 , предложенная модель неплохо описывает поведение коэффициентов распыления в пороговой области. Обращает на себя внимание то, что кривые для ионов близких масс практически совпадают. Имеется возможность экстраполирования данных на неизученные случаи.

\section{Финансирование работы}

Работа выполнена в рамках государственного задания Министерства науки и высшего образования РФ для Федерального государственного бюджетного учреждения науки Физико-технического института им. А.Ф. Иоффе Российской академии наук.

\section{Конфликт интересов}

Авторы заявляют, что у них нет конфликта интересов.

\section{Список литературы}

[1] Babenko P.Yu., Mironov M.I., Mikhailov V.S., Zinoviev A.N. // Plasma Phys. Control. Fusion. 2020. V. 62. P. 045020. DOI: 10.1088/1361-6587/ab7943

[2] Makarov S., Kaveeva E. // EECE-2018. MATEC Web Conf. 2018. V. 245. P. 13002.

DOI: $10.1051 /$ matecconf $/ 201824513002$

[3] Köchl F., Loarte A., de la Luna E., Parail V., Corrigan G., Harting D., Nunes I., Reux C., Rimini F.G., Polevoi A., Romanelli M. and JET Contributors // Plasma Phys. Control. Fusion. 2018. V. 60. P. 074008.

DOI: $10.1088 / 1361-6587 /$ aabf52 
[4] Abrams T., Unterberg E.A., Rudakov D.L., Leonard A.W., Schmitz O., Shiraki D., Baylor L.R., Stangeby P.C., Thomas D.M., Wang H.Q. // Phys. Plasmas. 2019. V. 26. P. 062504. DOI: $10.1063 / 1.5089895$

[5] Bobkov Vl., Bilato R., Braun F., Dux R., Herrmann A., Kallenbach A., Neu R., Noterdaeme J.M., Pütterich Th., and ASDEX Upgrade Team // AIP Conf. Proc. 2007. V. 933. P. 83-88. DOI: $10.1063 / 1.2800554$

[6] Brezinsek S. // J. Nucl. Mater. 2015. V. 463. P. 11-21. DOI: 10.1016/j.jnucmat.2014.12.007

[7] Keima A., Harnisch M., Scheier P., Herman Z. // Int. J. Mass Spectrom. 2013. V. 354-355. P. 78-86. DOI: $10.1016 /$ j.jims.2013.06.002

[8] Harnisch M., Keima A., Scheier P., Herman Z. // Int. J. Mass Spectrom. 2014. V. 365-366. P. 316-323. DOI: $10.1016 /$ j.ijms.2014.02.013

[9] Träskelin P., Juslin N., Erhart P., Nordlund K. // Phys. Rev. B. 2007. V. 75. P. 174113. DOI: 10.1103/PhysRevB.75.174113

[10] Kukushkin A.S., Pacher H.D., Kotov V., Pacher G.W., Reiter D. // Fusion Eng. Design. 2011. V. 86. P. 2865-2873. DOI: 10.1016/j.fusengdes.2011.06.009

[11] Doerner R.P. // J. Vac. Sci. Technol. A. 2005. V. 23. P. $1545-$ 1547. DOI: $10.1116 / 1.2110385$

[12] Nogami K., Sakai Y., Mineta S., Kato D., Murakami I., Sakaue H.A., Kenmotsu T., Furuya K., Motohashi K. // J. Vac. Sci. Technol. A. 2015. V. 33. P. 061602. DOI: $10.1116 / 1.4928250$

[13] Hua X., He H., Ding W., Ding R., Chen J., Pan B. // Chin. J. Chem. Phys. 2017. V. 30. P. 77-82. DOI: $10.1063 / 1674-0068 / 30 /$ cjcp1607138

[14] Marinica M.-C., Ventelon L., Gilbert M.R., Proville L., Dudarev S.L., Marian J., Bencteux G., Willaime F. // J. Phys.: Condens. Matter. 2013. V. 25. P. 395502.

DOI: 10.1088/0953-8984/25/39/395502

[15] Sand A.E., Dequeker J., Becquart C.S., Domain C., Nordlund K. // J. Nucl. Mater. 2016. V. 470. P. 119-127. DOI: 10.1016/j.jnucmat.2015.12.012

[16] Meluzova D.S., Babenko P.Yu., Shergin A.P., Nordlund K., Zinoviev A.N. // Nucl. Instrum. Meth. Phys. Res. B. 2019. V. 460. P. 4-9. DOI: 10.1016/j.nimb.2019.03.037

[17] https://users.cecs.anu.edu.au/ u9300839/

[18] Behrisch R., Eckstein W. Sputtering by particle bombardment. Berlin: Springer, 2007. 526 p.

[19] Yamamura Y., Tawara H. // Atom. Data Nucl. Data Tabl. 1996. V. 62. P. 149-253. DOI: $10.1006 /$ adnd. 1996.0005

[20] Yamamura Y., Itikawa Y., Itoh $N$. Angular dependence of sputtering yields of monatomic solids. Report IPPJ-AM-26. Nagoya, 1983.

[21] Nakamura H., Saito S., Ito A.M. // J. Adv. Simulat. Sci. Eng. 2016. V. 3. P. $165-172$. DOI: $10.15748 /$ jasse.3.165

[22] Ziegler J.F., Biersack J.P. SRIM [Электронный pecypc]. Режим доступа: http://www.srim.org

[23] Yang X., Hassanein A. // Appl. Surf. Sci. 2014. V. 293. P. 187190. DOI: $10.1016 /$ j.apsusc.2013.12.129

[24] Eckstein $W$. Sputtering, reflection and range values for plasma edge codes. IPP report 9/117. Garching, 1998. 